\title{
Cross-Electrophile Coupling of Alcohols with Aryl and Vinyl Halides
}

\author{
Benjamin K. Chi, Jonas K. Widness, Michael M. Gilbert, Daniel C. Salgueiro, Kevin J. Garcia, Daniel J. Weix*
}

University of Wisconsin-Madison, Department of Chemistry, 1101 University Ave, Madison, WI 53706

Supporting Information Placeholder

ABSTRACT: Although alcohols represent one of the largest pools of commercially available alkyl substrates, approaches to directly utilize them in cross-coupling and crosselectrophile coupling are limited. We report the use of alcohols in cross-electrophile coupling with aryl and vinyl halides to form $\mathrm{C}\left(\mathrm{sp}^{3}\right)-\mathrm{C}\left(\mathrm{sp}^{2}\right)$ bonds in a one-pot strategy. This strategy allows the use of primary and secondary alcohols through their very fast $(<1 \mathrm{~min})$ in situ conversion to the corresponding alkyl bromides with compatible phosphonium activating reagents. The utility of the reaction is exemplified by its simple reaction setup, scalability, and broad scope (41 examples, $57 \% \pm 15 \%$ ave yield). The reaction can be performed on the benchtop without the need for electrochemical or photochemical equipment. Finally, translation to standard parallel synthesis techniques is demonstrated by successfully coupling all combinations of 8 alcohols with 12 aryl cores in a 96-well plate using only one (99\% coverage) or two (100\% coverage) sets of conditions.

Cross-coupling reactions to form $\mathrm{C}\left(\mathrm{sp}^{3}\right)-\mathrm{C}\left(\mathrm{sp}^{2}\right)$ bonds are increasingly important for the synthesis of structurally diverse molecules in medicinal chemistry ${ }^{1}$ and natural product synthesis. $^{2}$ In medicinal chemistry, small-scale highthroughput experimentation is now routine to allow rapid synthesis of focused libraries to explore structure-activity relationships (SAR) and optimize lead compounds while preserving valuable material. ${ }^{3}$ In these studies, the size of the substrate pool is crucial and even one-step activation approaches that require intermediate isolation or purification can be limiting (Figure 1a). Alcohols have long been the primary source of alkyl diversity in cross-coupling via their conversion to alkyl halides, sulfonate esters, ${ }^{4}$ and (more recently) other redox-activated derivatives. ${ }^{5}$ Conversion of alcohols to halides and pseudohalides represents the most-used functional group interconversion in medicinal chemistry, ${ }^{1 \mathrm{a}, 6}$ however such reactions require additional time and resources for each synthesis and purification, imposing an inherent bottleneck in the conversion of alcohols to libraries of pharmaceutical compounds (Figure 1b). General one-pot activa- tion/coupling strategies compatible with high-throughput experimentation (HTE) approaches ${ }^{7}$ have been elusive and largely limited to activated alcohols. ${ }^{8}$

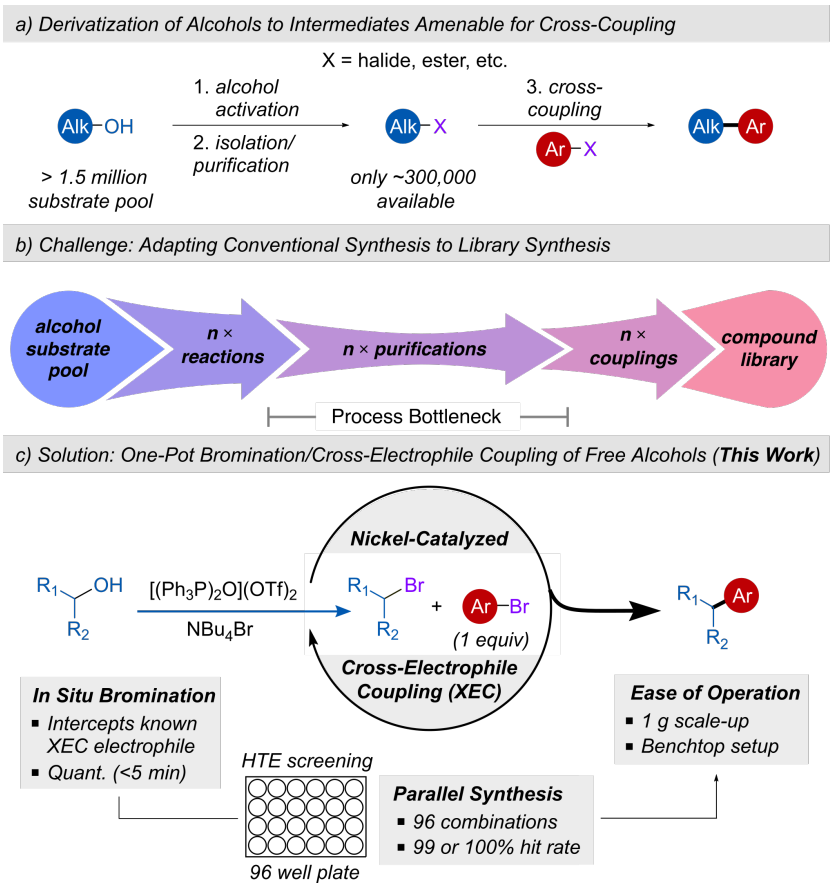

Figure 1. Direct Cross-Electrophile Coupling of Alcohols with Aryl Bromides Via In Situ Bromination.

A direct, yet under-explored, approach would be the in-situ conversion of alcohols to alkyl bromides in tandem with cross-electrophile coupling (XEC). ${ }^{9,10}$ While building upon established chemistry, this approach presents several challenges. First, the activating agent must be selective for the target alcohol over other Lewis-basic functionalities (e.g., amides, ketones) and not directly react with other components of the reaction (catalyst, reductant, solvent). Second, the co-products of this activation (e.g., phosphine oxide, acid) must be tolerated by the coupling reaction. Finally, the reaction must be fast and high yielding to avoid the need for multiple manipulations of each reaction (Figure $1 \mathrm{~b}$ and $1 \mathrm{c}$ ).

We have found that employing oxophilic $\mathrm{P}(\mathrm{V})$ reagents, especially Hendrickson's POP reagent $\left(\left[\left(\mathrm{Ph}_{3} \mathrm{P}\right)_{2} \mathrm{O}\right](\mathrm{OTf})_{2}\right),{ }^{11}$ for one-pot alcohol activation is 
compatible with XEC conditions, procedurally simple, general, fast, and well-suited to $\mu$ mol-scale HTE format (Figure 1c). Concurrent with these studies, two other exciting advances towards this goal have appeared that utilize paired electrolysis ${ }^{10}$ and metallaphotoredox catalysis. ${ }^{12}$ While all three approaches are impressively general, our approach appears better suited to parallel synthesis, ${ }^{13,14}$ does not require excess of either coupling partner to achieve selective crosscoupling, and requires no specialized electrochemical or photochemical equipment for preparative or HTE applications.

Keys to the success of this approach are: 1) the discovery of a fast, homogeneous bromination reagent, $\mathrm{POP} / \mathrm{TBAB}$ $\left(\mathrm{Bu}_{4} \mathrm{NBr}\right)$ in $\mathrm{MeCN}$, that is compatible with reductive coupling conditions; and 2) the development of a new ligand, 4,4'-di-tert-butyl-6- $\mathrm{N}$-cyanocarboxamidine-2,2-bipyridine ( ${ }^{t}$ ${ }^{\mathrm{Bu} B p y C a m}{ }^{\mathrm{CN}}$ or L1) that, alone or in combination with dtbbpy, ${ }^{15}$ provides high yields of product. Because the new catalyst mixture works well in acetonitrile, problematic amide solvents can be avoided ${ }^{16}$ and the bromination can occur concurrent with the cross-electrophile coupling (Table 1).

A significant practical advantage of our approach is the use of a POP/TBAB reagent solution in $\mathrm{MeCN}$, which can be easily handled outside of a glovebox and facilitates parallel reaction assembly. While amide solvents appeared better at solubilizing this reagent and are established for crosselectrophile coupling, we obtained low yields in DMA (Table 1 , entry 15) and POP reagents have been reported to be reactive with amides. ${ }^{17}$ The tetrabutylammonium cation is critical for the solubility of the POP reagent in acetonitrile as other cations resulted in slurries that would be impractical on small scale (see Supplementary Table S3). The solution is stable for months when stored in a septum vial in a desiccator without any decrease in reactivity (entry 8 ). Compared to triphenylphosphine dibromide (the intermediate invoked in the paired electrolysis report ${ }^{10}$ ), we found bromination with $\mathrm{POP} / \mathrm{TBAB}$ faster (complete in $5 \mathrm{~min}$ at $\mathrm{rt}$ ). In practice this allowed us to run reactions without any monitoring of the bromination.

Control reactions showed that each component of the system is necessary for high yields (Table 1, entry 2-5). Triphenylphosphine dibromide and $\left[\left(\mathrm{Me}_{3} \mathrm{P}\right)_{2} \mathrm{O}\right](\mathrm{OTf})_{2} / \mathrm{TBAB}$ were both reasonable alternatives to POP (Table 1, entries 6 and 7). Triphenylphosphine dibromide proved useful with substrates prone to elimination, ${ }^{18}$ (3ag, Scheme 1) and $\left.\left(\mathrm{Me}_{3} \mathrm{P}\right)_{2} \mathrm{O}\right](\mathrm{OTf})_{2}$ could be useful on larger scale because $\mathrm{Me}_{3} \mathrm{P}=\mathrm{O}$ is water soluble. While a variety of nonnucleophilic organic bases were effective, Barton's base $\left(\left(\mathrm{Me}_{2} \mathrm{~N}\right)_{2} \mathrm{C}=\mathrm{N}(t-\mathrm{Bu})\right)$ was superior (entries 1, 9, Supplementary Table S2). The reactions worked best with an excess of Mn (entries 1, 12-13), but could be run at lower temperature and without a glovebox, if needed (entries 14, 8).

Informed by our previous studies on carboxamidine ligands ${ }^{19}$ and mixed-ligand systems, ${ }^{15}$ we found that a new mixed-ligand system with ${ }^{t b u}$ BpyCam ${ }^{\mathrm{CN}}(\mathbf{L 1})^{20}$ and dtbbpy provided enhanced selectivity for the cross-coupled product in comparison to a single ligand system (Table 1, entry 9-10 and Supporting Information Table S1). Examination of our results with single ligands showed that $\mathbf{L 1}$ and dtbbpy had complementary reactivity: dtbbpy-ligated Ni primarily consumed the aryl bromide, whereas L1-ligated $\mathrm{Ni}$ favored alkyl bromide consumption. The synergistic effect of both ligands enabled the development of a general, tunable reaction system (vide infra, Scheme 2), even in a non-amide solvent. ${ }^{21}$

Table 1. Optimization of Deoxygenative Cross-Electrophile Coupling with Aryl Bromides. ${ }^{a}$

$\mathrm{NiBr}_{2} \mathrm{dme}(10 \mathrm{~mol} \%)$
$\mathrm{LOP}(1$ mol\%), dtbby (5 mol\%)
$\mathrm{Barton}$ 's base (1 equiv)

${ }^{a}$ Reactions run at a $0.25 \mathrm{mmol}$ scale. The alcohol, $\mathrm{POP} / \mathrm{TBAB}$ solution, and base were mixed for $30 \mathrm{~s}$ before being combined with the rest of the reagents. ${ }^{b}$ Corrected GC yields. Isolated yields shown in parentheses. ${ }^{c}$ Reaction run with $\mathrm{POP} / \mathrm{TBAB}$ solution stored in a desiccator for 2 months.

These new conditions enable a simple, one-pot reaction for the cross-coupling of alcohols with aryl bromides by stepwise addition of reagents. The alcohol is combined with the POP/TBAB solution and Barton's base for about $1 \mathrm{~min}$ before being combined with the catalyst, aryl bromide, and Mn. Importantly, the alcohol activation solution remains 
homogeneous throughout, with no need to monitor for completion.

This system proved effective for the coupling of a wide array of $1^{\circ}$ and $2^{\circ}$ unactivated alcohols with aryl, heteroaryl,

Scheme 1. Substrate Scope for the Deoxygenative Cross-Coupling of Alcohols with Aryl, Heteroaryl, and Vinyl Halides ${ }^{a}$

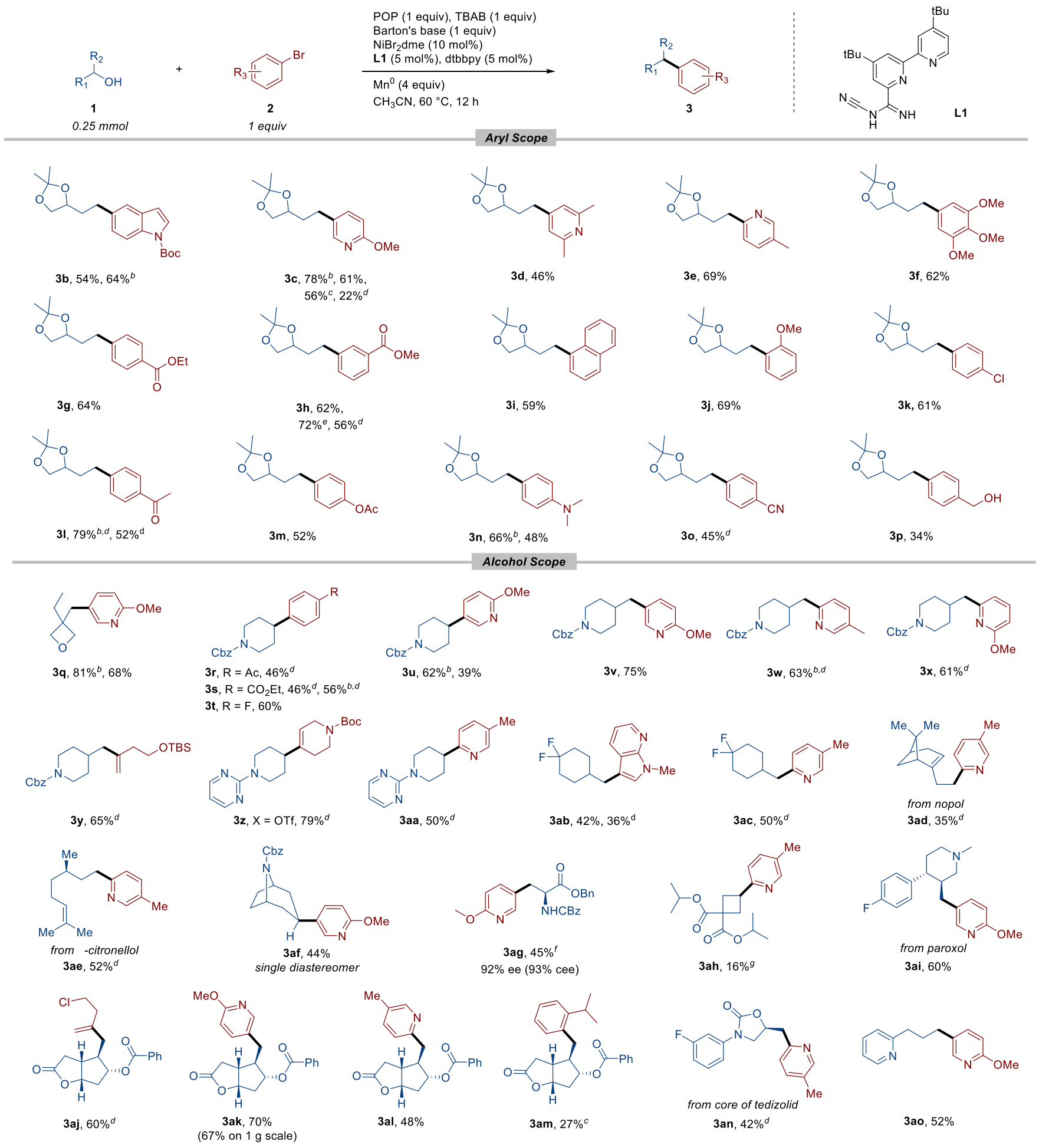

${ }^{a}$ Isolated yields of reactions run at $0.25 \mathrm{mmol}$ scale. Alcohol 1, POP/TBAB solution, and base were mixed for $1 \mathrm{~min}$ before being combined with the rest of the reagents, capped, and stirred at $60^{\circ} \mathrm{C}$ overnight. ${ }^{b} 1.5$ equiv of alcohol, POP, TBAB, and Barton's base used. ${ }^{c}$ Only dtbbpy $(10 \mathrm{~mol} \%)$ was used. ${ }^{d}$ Only $\mathbf{L} \mathbf{1}(10 \mathrm{~mol} \%)$ was used. ${ }^{e}(1: 3) \mathrm{dtbbpy}: \mathbf{L} \mathbf{1}$ used. ${ }^{f} 1.25$ equiv of $\mathrm{PPh}_{3} \mathrm{Br}_{2}$, and

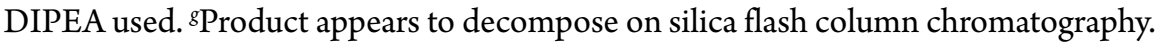


Aryl substrates bearing esters (3g-h, 3m, 3s), ethers (3c, 3f,

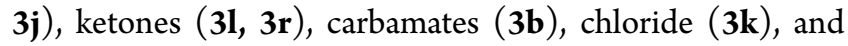
heteroarenes (3b-e) were compatible under these conditions. Sterically hindered aryl substrates bearing ortho substituents such as in 2-naphthalene, methoxy, and isopropyl could also be coupled (3i-j, 3am). Because the alcohol activation is usually complete before aryl bromide is added, the development of reactions that tolerate free alcohols on the aryl bromide is feasible: in the coupling of $\mathbf{3 p}$ the less reactive alcohol is coupled over the benzylic alcohol, albeit in only $34 \%$ yield (unoptimized). Future improvements will enable telescoped three-component reactions and sequential crosscouplings.

While the optimized two-ligand system worked best for electron-rich aryl halides, more reactive $\mathrm{C}\left(\mathrm{sp}^{2}\right)$ electrophiles, such as 2-bromopyridines (3w, 3x, 3aa, 3ac, 3ad, 3ae, 3an), aryl bromides with strong electron withdrawing groups in the para position $(\mathbf{3 u}, \mathbf{3 v})$, and alkenyl electrophiles $(\mathbf{3 y}, \mathbf{3 z}$, 3aj) performed best using only L1 (10 $\mathrm{mol} \%$ ) with no dtbbpy. Furthermore, the ratio of $\mathbf{L} \mathbf{1}$ to dtbbpy can be adjusted to improve yields: the coupling between primary alcohol $\mathbf{1 b}$ and methyl 3-bromobenzoate $\mathbf{2 h}$ afforded the product in $72 \%$ yield with $75: 25 \mathrm{L1} /$ dtbbpy compared to $62 \%$ using a 1:1 ligand ratio.

We next explored the scope of the alcohol coupling partner. Our attention was focused on alcohol substrates for which the corresponding alkyl bromide was either not commercially available or is an order of magnitude more expensive per mole (Supporting Information Table S3). A wide variety of primary and secondary alcohols ${ }^{23}$ could be coupled with aryl, heteroaryl, and vinyl substrates to give a structurally diverse set of products. Finally, we could scale the reaction of 3ak from $0.25 \mathrm{mmol}$ to $3.6 \mathrm{mmol}$ scale using standard glassware with about the same yield (from $70 \%$ to $67 \%$ yield).

\section{Scheme 2. Coupling of Alcohols with Aryl Halides on $10 \mu \mathrm{mol}$ Scale in 96-Well Plate Format.}

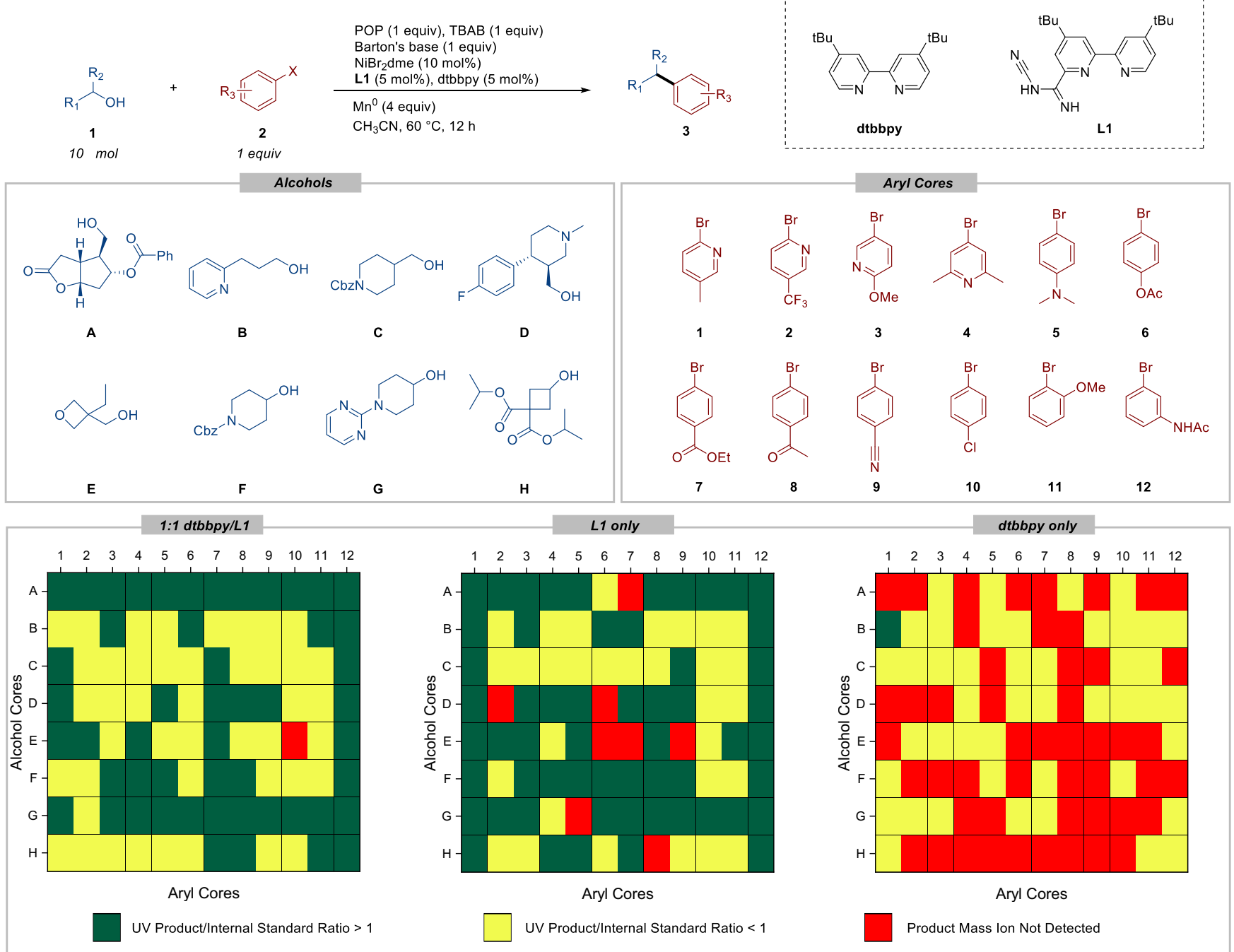

${ }^{a} \mathrm{HTE}$ survey of 96 combinations of alcohols and aryl bromides with each reaction run at $10 \mu \mathrm{mol}$ scale. UV Product/Internal standard $\begin{array}{lllll}\text { ratios } & \text { vs. } & \text { 1,3,5-trimethoxybenzene } & \text { fbtained }\end{array}$ 
More hindered alcohols and those with $\beta$-heteroatoms reacted more slowly and were prone to form elimination products, consistent with literature reports on the POP reagent. ${ }^{18}$ A preliminary workaround is the use of $\mathrm{Ph}_{3} \mathrm{PBr}_{2}$ instead of $\mathrm{POP} / \mathrm{TBAB}$, which enabled coupling with serine to form heteroaryl phenylalanine derivative 3 ag with useful stereoretention ( $93 \%$ cee).

As noted above, the ability to directly use alcohols in crosselectrophile coupling reactions without extra steps will be of particular advantage in the generation of small libraries in medicinal chemistry. ${ }^{1,19 c}$ To explore this application, we coupled all combinations of 12 aryl halide cores with 8 alcohols on $10 \mu \mathrm{mol}$ scale in a 96 well plate using three ligand regimes (dtbbpy only, 1:1 dtbbpy/L1, and $\mathbf{L} 1$ only). All reagents except $\mathrm{Mn}$ were dosed using liquid handling and standard multichannel pipettes. We used the AbbVie Mn@Chembead approach $^{19 c}$ to dose the Mn using a calibrated scoop and a shaker/heater was used in place of a tumble stirrer. These results show that 1:1 dtbbpy/L1 is the most general set of conditions (product observed in 95/96 cases, 99\% hit rate), but that $\mathbf{L} \mathbf{1}$ alone can improve yields for some combinations: E10 had no detected product with $\mathbf{L 1} /$ dtbbpy but product was detected with $\mathbf{L} \mathbf{1}$ alone. When combined, the two catalyst combinations, dtbbpy/L1 and $\mathbf{L 1}$, provided product in all 96 combinations.

Mechanistically, the bromination and cross-electrophile coupling reactions are expected to proceed by their established mechanisms. ${ }^{24}$ The bromination reaction is quantitative in about $5 \mathrm{~min}$ at $\mathrm{rt}$ (monitored by GC). Nickelmediated cross-electrophile coupling then occurs between the alkyl bromide and the aryl electrophile.

In conclusion, the combination of a new catalyst system (dtbbpy/L1) and a carefully-tuned bromination reagent (POP/TBAB/Barton's base) enables a new, general crosselectrophile coupling of unactivated $1^{\circ}$ and $2^{\circ}$ alcohols with aryl bromides. The power of this approach is exemplified by the fact that 36 out of the 40 products in Scheme 1 are new compounds, despite being simple derivatives of commercial materials. Further, because the alcohol activation reagents and byproducts of the bromination step are homogeneous and do not impede cross-electrophile coupling step, these conditions are ideal for parallel synthesis. This advance, in tandem with related advances from MacMillan ${ }^{5}$ and $\mathrm{Li}^{9}{ }^{9}$ may lead to alcohols supplanting alkyl halides in the synthesis of $\mathrm{Csp}^{3}-\mathrm{Csp}^{2}$ bonds. Further, these reactions set the stage for selective deoxygenation of more complex polyols, such as those recently reported by Diao ${ }^{5 c}$ and MacMillan, ${ }^{12}$ and improved activating agents tuned to cover an even wider range of alcohols.

\section{ASSOCIATED CONTENT}

\section{Supporting Information.}

The Supporting Information is available free of charge on the ACS Publications website. additional tables of optimization data, experimental procedures, and characterization data for all isolated compounds (PDF)

\section{AUTHOR INFORMATION}

\section{Corresponding Author}

*dweix@wisc.edu

Daniel J. Weix - Department of Chemistry, University of Wisconsin-Madison, Madison, Wisconsin 53706, United States

\section{Authors}

Benjamin K. Chi - Department of Chemistry, University of Wisconsin-Madison, Madison, Wisconsin 53706, United States Jonas K. Widness - Department of Chemistry, University of Wisconsin-Madison, Madison, Wisconsin 53706, United States Michael M. Gilbert - Department of Chemistry, University of Wisconsin-Madison, Madison, Wisconsin 53706, United States Daniel C. Salgueiro - Department of Chemistry, University of Wisconsin-Madison, Madison, Wisconsin 53706, United States Kevin J. Garcia - Department of Chemistry, University of Wisconsin-Madison, Madison, Wisconsin 53706, United States

\section{ACKNOWLEDGMENTS}

This work was supported by the NIH (R01GM097243). The following instrumentation in the PBCIC was supported as follows: Thermo Q Extractive Plus by NIH 1S10 OD020022; Shimadzu GCMS-QP2010S by the Department of Chemistry; Bruker Avance III 400 by NSF CHE-1048642; Bruker Avance III 500 by a generous gift from Paul J. and Margaret M. Bender. We thank Nathan Loud (UW-Madison) for assistance with HTE and Kai Kang (UW-Madison) for assistance editing the manuscript.

\section{REFERENCES}

(1) (a) Dombrowski, A. W.; Gesmundo, N. J.; Aguirre, A. L.; Sarris, K. A.; Young, J. M.; Bogdan, A. R.; Martin, M. C.; Gedeon, S.; Wang, Y. Expanding the Medicinal Chemist Toolbox: Comparing Seven C(Sp2)$\mathrm{C}(\mathrm{Sp} 3)$ Cross-Coupling Methods by Library Synthesis. ACS Med. Chem. Lett. 2020, 11, 597-604. (b) Beutner, G. L.; Simmons, E. M.; Ayers, S.; Bemis, C. Y.; Goldfogel, M. J.; Joe, C. L.; Marshall, J.; Wisniewski, S. R. A Process Chemistry Benchmark for Sp2-Sp3 Cross Couplings. J. Org. Chem. 2021, 86, 10380-10396.

(2) (a) Tabassum, S.; Zahoor, A. F.; Ahmad, S.; Noreen, R.; Khan, S. G.; Ahmad, H. Cross-Coupling Reactions towards the Synthesis of Natural Products. Mol. Diversity 2021. (b) Fujii, S.; Chang, S. Y.; Burke, M. D. Total Synthesis of Synechoxanthin through Iterative Cross-Coupling. Angew. Chem. Int. Ed. 201 1, 50, 7862-7864. (c) Cheng, H.-G.; Yang, Z.; Chen, R.; Cao, L.; Tong, W.-Y.; Wei, Q.; Wang, Q.; Wu, C.; Qu, S.; Zhou, Q. A Concise Total Synthesis of (-)-Berkelic Acid. Angew. Chem. Int. Ed. 2021, 60, 5141-5146. (d) Yan, B.-C.; Zhou, M.; Li, J.; Li, X.-N.; He, S.-J.; Zuo, J.-P.; Sun, H.-D.; Li, A.; Puno, P.-T. (-)-Isoscopariusin A, a Naturally Occurring Immunosuppressive Meroditerpenoid: Structure Elucidation and Scalable Chemical Synthesis. Angew. Chem. Int. Ed. 2021, 60, 12859-12867. (e) Luo, L.; Zhai, X.-Y.; Wang, Y.-W.; Peng, Y.; Gong, H. Divergent Total Syntheses of C3 A-C7' Linked Diketopiperazine Alkaloids (+)-Asperazine and (+)-Pestalazine A Enabled by a NiCatalyzed Reductive Coupling of Tertiary Alkyl Chloride. Chem -Eur. J. 2019, 25, 989-992. 
(3) Mennen, S. M.; Alhambra, C.; Allen, C. L.; Barberis, M.; Berritt, S.; Brandt, T. A.; Campbell, A. D.; Castañón, J.; Cherney, A. H.; Christensen, M.; Damon, D. B.; Eugenio de Diego, J.; García-Cerrada, S.; GarcíaLosada, P.; Haro, R.; Janey, J.; Leitch, D. C.; Li, L.; Liu, F.; Lobben, P. C.; MacMillan, D. W. C.; Magano, J.; McInturff, E.; Monfette, S.; Post, R. J.; Schultz, D.; Sitter, B. J.; Stevens, J. M.; Strambeanu, I. I.; Twilton, J.; Wang, K.; Zajac, M. A. The Evolution of High-Throughput Experimentation in Pharmaceutical Development and Perspectives on the Future. Org. Process Res. Dev. 2019, 23, 1213-1242.

(4) Molander, G. A.; Traister, K. M.; O’Neill, B. T. Engaging Nonaromatic, Heterocyclic Tosylates in Reductive Cross-Coupling with Aryl and Heteroaryl Bromides. J. Org. Chem. 2015, 80, 2907-2911.

(5) (a) Zhang, X.; MacMillan, D. W. C. Alcohols as Latent Coupling Fragments for Metallaphotoredox Catalysis: Sp3-Sp2 Cross-Coupling of Oxalates with Aryl Halides. J. Am. Chem. Soc. 2016, 138, 1386213865. (b) Lackner, G. L.; Quasdorf, K. W.; Overman, L. E. Direct Construction of Quaternary Carbons from Tertiary Alcohols via Photoredox-Catalyzed Fragmentation of Tert-Alkyl N-Phthalimidoyl Oxalates. J. Am. Chem. Soc. 2013, 135, 15342-15345. (c) Wei, Y.; Ben-zvi, B.; Diao, T. Diastereoselective Synthesis of Aryl C-Glycosides from Glycosyl Esters via C-O Bond Homolysis. Angew. Chem. Int. Ed. 2021, 60, 94339438. (d) Kariofillis, S.; Jiang, S.; Żurański, A.; Gandhi, S.; Martinez Alvarado, J.; Doyle, A. ChemRxiv 2021. This content is a preprint and has not been peer-reviewed. doi: 10.33774/chemrxiv-2021-6kd0t https://doi.org/10.33774/chemrxiv-2021-6kd0t [Accessed October 16, 2021]. (e) Gao, M.; Sun, D.; Gong, H. Ni-Catalyzed Reductive C-O Bond Arylation of Oxalates Derived from $\alpha$-Hydroxy Esters with Aryl Halides. Org. Lett. 2019, 21, 1645-1648. (f) Ye, Y.; Chen, H.; Sessler, J. L.; Gong, H. Zn-Mediated Fragmentation of Tertiary Alkyl Oxalates Enabling Formation of Alkylated and Arylated Quaternary Carbon Centers. J. Am. Chem. Soc. 2019, 141, 820-824.

(6) (a) Carey, J. S.; Laffan, D.; Thomson, C.; Williams, M. T. Analysis of the Reactions Used for the Preparation of Drug Candidate Molecules. Org. Biomol. Chem. 2006, 4, 2337-2347. (b) Roughley, S. D.; Jordan, A. M. The Medicinal Chemist's Toolbox: An Analysis of Reactions Used in the Pursuit of Drug Candidates. J. Med. Chem. 2011, 54, 3451-3479. (c) Schneider, N.; Lowe, D. M.; Sayle, R. A.; Tarselli, M. A.; Landrum, G. A. Big Data from Pharmaceutical Patents: A Computational Analysis of Medicinal Chemists' Bread and Butter. J. Med. Chem. 2016, 59, 43854402.

(7) It is widely understood that one-pot procedures which bypass isolation and purification steps increase the efficiency of chemical synthesis with respect to time, waste production, and ultimately cost. With solvent use constituting $80-90 \%$ of material mass (not including water) in API synthesis, one-pot activation/cross coupling protocols without intermediate processing (e.g. solvent exchange, filtration, etc.) represent a powerful approach to conserving both time and resources, especially in the context of HTE. See the following references: (a) Hayashi, Y. Pot Economy and One-Pot Synthesis. Chem. Sci. 2016, 7, 866-880. (b) Vaxelaire, C.; Winter, P.; Christmann, M. One-Pot Reactions Accelerate the Synthesis of Active Pharmaceutical Ingredients. Angew. Chem. Int. Ed. 2011, 50, 3605-3607.

(8) (a) Suga, T.; Ukaji, Y. Nickel-Catalyzed Cross-Electrophile Coupling between Benzyl Alcohols and Aryl Halides Assisted by Titanium Co-Reductant. Org. Lett. 2018, 20, 7846-7850. (b) Ackerman, L. K. G.; Anka- Lufford, L. L.; Naodovic, M.; Weix, D. J. Cobalt co-catalysis for cross- electrophile coupling: diarylmethanes from benzyl mesylates and aryl halides. Chem. Sci. 2015, 6, 1115-1119. (c) Kuwano, R.; Yokogi, M. Cross-Coupling of Benzylic Acetates with Arylboronic Acids: One-Pot Transformation of Benzylic Alcohols to Diarylmethanes. Chem. Commun. 2005, 5899. (d) Guo, P.; Wang, K.; Jin, W.-J.; Xie, H.; Qi, L.; Liu, X.-Y.; Shu, X.-Z. Dynamic Kinetic Cross-Electrophile Arylation of Benzyl Alcohols by Nickel Catalysis. J. Am. Chem. Soc. 2021, 143, 513-523. (e) Ma, W.-Y.; Han, G.-Y.; Kang, S.; Pang, X.; Liu, X.-Y.; Shu, X.-Z. Co-
balt-Catalyzed Enantiospecific Dynamic Kinetic Cross-Electrophile Vinylation of Allylic Alcohols with Vinyl Triflates. J. Am. Chem. Soc. 2021, 143, 15930-15935. (f) Yu, D.-G.; Wang, X.; Zhu, R.-Y.; Luo, S.; Zhang, X.-B.; Wang, B.-Q.; Wang, L.; Shi, Z.-J. Direct Arylation/Alkylation/Magnesiation of Benzyl Alcohols in the Presence of Grignard Reagents via Ni-, $\mathrm{Fe}-$, or Co-Catalyzed $\mathrm{sp}^{3} \mathrm{C}-\mathrm{O}$ Bond Activation. J. Am. Chem. Soc. 2012, 134, 14638-14641. (g) Cao, Z.-C.; Yu, D.G.; Zhu, R.-Y.; Wei, J.-B.; Shi, Z.-J. Direct Cross-Coupling of Benzyl Alcohols to Construct Diarylmethanes via Palladium Catalysis. Chem. Commun. 2015, 51, 2683-2686.

(9) Li, Z.; Sun, W.; Wang, X.; Li, L.; Zhang, Y.; Li, C. Electrochemically Enabled, Nickel-Catalyzed Dehydroxylative Cross-Coupling of Alcohols with Aryl Halides. J. Am. Chem. Soc. 2021, 143, 3536-3543.

(10) One-pot conversion and coupling of amines, alcohols, and alkanoic acids have recently been reported: amines (a) Liao, J.; Basch, C. H.; Hoerrner, M. E.; Talley, M. R.; Boscoe, B. P.; Tucker, J. W.; Garnsey, M. R.; Watson, M. P., Deaminative Reductive Cross-Electrophile Couplings of Alkylpyridinium Salts and Aryl Bromides. Org. Lett. 2019, 21, 29412946. (b) Ni, S.; Li, C.-X.; Mao, Y.; Han, J.; Wang, Y.; Yan, H.; Pan, Y., Ni-catalyzed deaminative cross-electrophile coupling of Katritzky salts with halides via $\mathrm{C}-\mathrm{N}$ bond activation. Sci. $A d v$. 2019, 5, eaaw9516. alcohols see ref. 8b-d. alkanoic acids (d) Koyanagi, T.; Herath, A.; Chong, A.; Ratnikov, M.; Valiere, A.; Chang, J.; Molteni, V.; Loren, J. One-Pot Electrochemical Nickel-Catalyzed Decarboxylative $\mathrm{Sp}^{2}-\mathrm{Sp}^{3}$ CrossCoupling. Org. Lett. 2019, 21, 816-820.

(11) Hendrickson, J. B.; Schwartzman, S. M. Triphenyl Phosphine Ditriflate: A General Oxygen Activator. Tetrahedron Lett., 1975, 16, 277-280. (12) Dong, Z.; MacMillan, D. W. C. Metallaphotoredox-Enabled Deoxygenative Arylation of Alcohols. Nature 2021, Online Ahead of Print. doi: 10.1038/s41586-021-03920-6 https://doi.org/10.1038/s41586-02103920-6 [Accessed October 16, 2021].

(13) Neither report details an adaptation to small scale parallel format and there appear to be barriers in both cases: a need for separate 15 min + activation steps + filtration of every alcohol ${ }^{12}$ or a reliance on parallel electrochemical equipment that is not yet generally available. ${ }^{10}$

(14) While photoredox catalysis has been adapted to parallel synthesis, parallel electrochemistry is still nascent. See: Rein, J.; Annand, J. R.; Wismer, M. K.; Fu, J.; Siu, J. C.; Klapars, A.; Strotman, N. A.; Kalyani, D.; Lehnherr, D.; Lin, S. Unlocking the Potential of High-Throughput Experimentation for Electrochemistry with a Standardized Microscale Reactor. ACS Cent. Sci. 2021, 7, 1347-1355

(15) Mixtures of nickel catalysts generally help tune the rate of alkyl radical generation and have been repeatedly been found to be useful in cross-electrophile coupling reactions. See: (a) Everson, D. A.; Shrestha, R.; Weix, D. J. Nickel-Catalyzed Reductive Cross-Coupling of Aryl Halides with Alkyl Halides. J. Am. Chem. Soc. 2010, 132, 920-921. (b) Perkins, R. J.; Hughes, A. J.; Weix, D. J.; Hansen, E. C. Metal-ReductantFree Electrochemical Nickel-Catalyzed Couplings of Aryl and Alkyl Bromides in Acetonitrile. Org. Process Res. Dev. 2019, 23, 1746-1751.

(16) (a) Alder, C. M.; Hayler, J. D.; Henderson, R. K.; Redman, A. M.; Shukla, L.; Shuster, L. E.; Sneddon, H. F. Updating and Further Expanding GSK's Solvent Sustainability Guide. Green Chem. 2016, 18, 38793890. (b) Prat, D.; Pardigon, O.; Flemming, H.-W.; Letestu, S.; Ducandas, V.; Isnard, P.; Guntrum, E.; Senac, T.; Ruisseau, S.; Cruciani, P.; Hosek, P. Sanofi's Solvent Selection Guide: A Step Toward More Sustainable Processes. Org. Process Res. Dev. 2013, 17, 1517-1525.

(17) POP has been reported to activate amides: Loughlin, W. A.; Jenkins, I. D.; Petersson, M. J. Cyclodehydration of N(Aminoalkyl)Benzamides under Mild Conditions with a Hendrickson Reagent Analogue. J. Org. Chem. 2013, 78, 7356-7361.

(18) Elson, K. E.; Jenkins, I. D.; Loughlin, W. A. The Hendrickson Reagent and the Mitsunobu Reaction: A Mechanistic Study. Org. Biomol. Chem. 2003, 1, 2958-2965. 
(19) (a) Hansen, E. C.; Pedro, D. J.; Wotal, A. C.; Gower, N. J.; Nelson, J. D.; Caron, S.; Weix, D. J. New ligands for nickel catalysis from diverse pharmaceutical heterocycle libraries. Nat. Chem. 2016, 8, 1126-1130. (b) Hansen, E. C.; Li, C.; Yang, S.; Pedro, D.; Weix, D. J. Coupling of Challenging Heteroaryl Halides with Alkyl Halides via Nickel-Catalyzed Cross-Electrophile Coupling. J. Org. Chem. 2017, 82, 7085-7092. (c) Aguirre, A. L.; Loud, N. L.; Johnson, K. A.; Weix, D. J.; Wang, Y. ChemBead Enabled High-Throughput Cross-Electrophile Coupling Reveals a New Complementary Ligand. Chem.-Eur. J. 2021, 27, 12981-12986.

(20) Ligand $\mathbf{L} \mathbf{1}$ is a new bipyridine carboxamidine derivative that can be synthesized on a $50 \mathrm{~g}$ scale in three steps from dtbbpy. It should soon be commercially available.

(21) Anka-Lufford, L. L.; Huihui, K. M. M.; Gower, N. J.; Ackerman, L. K. G.; Weix, D. J. Nickel-Catalyzed Cross-Electrophile Coupling with
Organic Reductants in Non-Amide Solvents. Chem.-Eur. J. 2016, 22, 11564-11567.

(22) For substrates that provided $<25 \%$ yield under Conditions A and B that have not yet been further optimized, see Supporting Information.

(23) We did not examine tertiary alcohols because the cross-electrophile coupling of tertiary radicals usually requires different conditions and several methods had already been reported for these alcohols. See reference $5 f$.

(24) (a) Biswas, S.; Weix, D. J. Mechanism and Selectivity in NickelCatalyzed Cross-Electrophile Coupling of Aryl Halides with Alkyl Halides. J. Am. Chem. Soc. 2013, 135, 16192-16197. (b) Diccianni, J. B.; Diao, T. Mechanisms of Nickel-Catalyzed Cross-Coupling Reactions. Trends Chem. 2019, 1, 830-844.

\section{TOC Graphic}

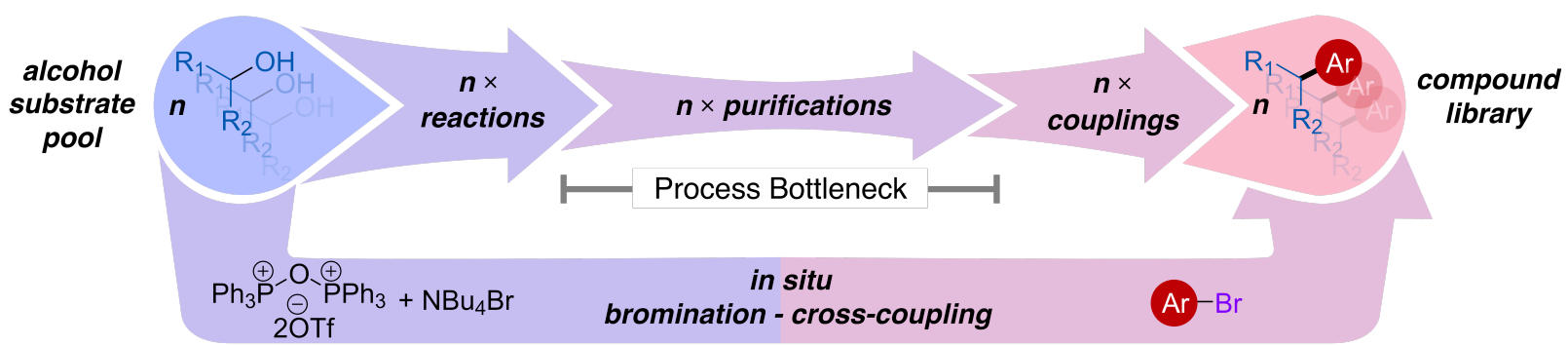

